\title{
Estructura de macroinvertebrados acuáticos y grupos funcionales tróficos en la cuenca del río Lluta, desierto de Atacama, Arica y Parinacota, Chile
}

\author{
Structure of aquatic macroinvertebrates and trophic functional groups \\ in the basin of Lluta river Atacama Desert, Arica y Parinacota, Chile
}

\author{
Marcos Ferrú ${ }^{1 *}$, Pablo Fierro ${ }^{2,3}$
}

\begin{abstract}
RESUMEN
El objetivo de este estudio fue determinar la estructura comunitaria de macroinvertebrados dulceacuícolas y caracterizar los grupos funcionales tróficos en la cuenca del río Lluta ubicado en el desierto de Atacama. Para esto se muestreó con una red Surber en 12 estaciones, abarcando desde la cabecera del río hasta su desembocadura en la costa marítima durante noviembre de 2011. En este estudio se registraron 66 taxa de macroinvertebrados, correspondiendo en su mayoría a larvas de insectos (46). Los grupos con mayor riqueza fueron Diptera (20 taxa), Coleoptera y Trichoptera (ambos con 7 taxa). Respecto de la abundancia, Chironomidae fue la familia más abundante del área de estudio. El grupo de los colectores-recolectores fue el más representativo en 10 de las 12 estaciones, mientras que el grupo de los ramoneadores dominaron en las dos estaciones más cercanas a la desembocadura. Nuestros resultados son los primeros en describir la fauna de macroinvertebrados dulceacuáticos en la cuenca más extrema del norte de Chile, conteniendo una riqueza faunística mucho mayor que otras cuencas desérticas. Además la gran representatividad de los colectores-recolectores que se alimentan de fragmentos orgánicos menores a $1 \mathrm{~mm}$ depositados en el fondo, demuestran la importancia de este recurso alimentario en ríos del desierto de Atacama.
\end{abstract}

Palabras clave: Macroinvertebrados acuáticos, grupos funcionales tróficos, Atacama, río Lluta, Chile.

\begin{abstract}
The aim of this study was to determine the community structure of freshwater macroinvertebrates and characterize trophic functional groups in the Lluta river basin located in the Atacama Desert. To this was sampled with Surber sampling in 12 stations, in extension from the source to its mouth on the coast during November 2011. In this study 66 taxa of macroinvertebrates were recorded, corresponding mostly insect larvae (46). The groups with the greatest wealth were Diptera (20 taxa), Coleoptera and Trichoptera (both with 7 taxa). On abundance, Chironomidae was the most abundant family of the study area. The group of collectors-gatherers was the most representative in 10 of the 12 stations, while the group of browsers dominated the two closest stations to the mouth. Our results are the first to describe the fauna of macroinvertebrates dulceacuáticos in extreme northern Chile basin, containing a much higher than other rich fauna desert basins. Besides the great representative of the collectors-gatherers that feed on organic fragments under $1 \mathrm{~mm}$ deposited on the bottom, showing the importance of this food source in the Atacama desert rivers.
\end{abstract}

Key words: Aquatics macroinvertebrates, trophic funtional groups, Atacama, Lluta river, Chile.

\section{Introducción}

En las últimas décadas, los múltiples usos que se les otorgan a los sistemas acuáticos continentales, e.g. consumo humano, turismo, actividades acuícolas, actividades mineras, etcétera, constituyen la principal causa del deterioro del hábitat, la calidad del agua y el funcionamiento de las comunidades asociadas, amenazando por tanto a su biodiversidad (Yixing et al. 2012). En Chile, las principales causas de la declinación de las poblaciones dulceacuícolas se debe a la pérdida o fragmentación del hábitat, a

\footnotetext{
Centro de Muestreo y Análisis Biológico (Cemabio), Valle Central \# 3240 Iquique-Chile Cemabio.cl.

Centro de Ciencias Ambientales EULA-CHILE, Universidad de Concepción, Concepción, Chile.

3 Instituto de Ciencias Marinas y Limnológicas, Facultad de Ciencias, Universidad Austral de Chile, Independencia 641, Valdivia, Chile.

* Autor para correspondencia: m.ferru@cemabio.cl
}

Fecha de Recepción: 3 Diciembre, 2014.

Fecha de Aceptación: 17 Agosto, 2015. 
la alteración de este por la urbanización y el uso de tecnología convencional en la agricultura que provocan contaminación en los cuerpos de agua, entre otros (Parada \& Peredo 2006).

En los ecosistemas dulceacuícolas, uno de los grupos más representativos son los macroinvertebrados bentónicos (Valdovinos 2006), los que consisten principalmente en estados inmaduros de insectos, ácaros, moluscos, crustáceos y anélidos, entre otros. Su distribución espacial ha sido relacionada con diversas variables que estructuran la comunidad a lo largo de los ríos, ya sean factores físicos, químicos o biológicos, como velocidad de la corriente, tipo y tamaño del sustrato, tamaño del río, niveles de descarga hidráulica, conductividad del agua, oxígeno disuelto e interacción con otras especies (Hynes 1970, Molina et al. 2008, Fierro et al. 2012). Factores que finalmente determinarán una gran diversidad de hábitats en los ríos, desde su cabecera hasta su desembocadura en el mar (Vannote et al. 1980).

Debido a la estrecha relación que hay en el ecotono de transición acuático-ribera, los cambios de uso de suelo tienen fuertes impactos sobre la biota, principalmente importando materia vegetal alóctona. Este material que penetra en los cuerpos acuáticos puede proveer de alimento a los invertebrados, y debido a que en general se presenta una zonación longitudinal de especies vegetales a lo largo de una cuenca, esta influiría en la variación de los grupos funcionales alimentarios (GFA) de macroinvertebrados (Miserendino \& Pizzolon 2003). De esta manera, generalmente se registran en la cabecera de las cuencas especies con hábitos fragmentadores, mientras que el río al llegar a la desembocadura con el mar (i.e. estuarios), abundan principalmente especies de naturaleza detritívora (Vannote et al. 1980, Fierro et al. 2014).

Las investigaciones de diversidad en poblaciones acuáticas que han sido llevadas a cabo en el norte de Chile son escasas, en especial en zonas áridas y semiáridas (Figueroa et al. 2009), por lo que la biodiversidad hasta ahora registrada estaría siendo subestimada. Esto queda reflejado en los recientes trabajos de Alvial et al. (2013) y Palma et al. (2013) en cuencas del desierto de Atacama, quienes citan 43 y 57 taxa de macroinvertebrados, un número bastante menor a lo registrado en otras cuencas ubicadas al sur de Atacama (Valdovinos et al. 2006).

La provincia de Atacama, perteneciente al bioma de la zona de transición de Sudamérica (Morrone
2006) presenta cuencas endorreicas y exorreicas, en donde la mayor parte de los ríos nacen en la cordillera de los Andes (desde los $5500 \mathrm{~m}$ de altura), en su camino recorren la Pampa, la cordillera de la Costa y finalmente desembocan en el océano Pacífico. Una de las cuencas más importantes en el desierto de Atacama es la cuenca del río Lluta, la que presenta el más importante humedal costero de la región, pues es un lugar fundamental de descanso de aves migratorias, siendo además el único río exorreico de la región, presentando como principal característica altas concentraciones de sales (sal, boro, sulfuros y sulfatos), las que son aportadas principalmente por los afluentes del curso superior (condiciones típicas de ecosistemas áridos y semiáridos) (Torres \& Acevedo 2008). Las mayores crecidas del caudal en estos ríos ocurren en verano, producto del invierno boliviano (Campos Ortega et al. 2007), las que provocan un gran efecto de erosión, arrastre, colmatación y sedimentación de sólidos suspendidos, un proceso dinámico que afecta al cauce principal en todo su recorrido, sometiendo por tanto a un periodo de estrés a las comunidades biológicas que allí se desarrollan.

Basado en estos antecedentes, el objetivo del presente estudio fue establecer la comunidad de macroinvertebrados dulceacuícolas que habitan la cuenca del río Lluta, y describir la heterogeneidad de estos por medio de los grupos funcionales alimentarios a lo largo del sistema, desde la cabecera del río hasta su desembocadura en el mar.

\section{Materiales y métodos}

\section{Sitio de estudio}

El sitio de estudio comprende toda la cuenca del río Lluta, ubicada en el extremo norte de Chile, en la XV Región de Arica y Parinacota, entre los paralelos $18^{\circ} 00^{\prime}-18^{\circ} 30^{\prime}$ latitud sur y meridianos $70^{\circ} 20^{\prime}-69^{\circ} 22^{\prime}$ ' longitud oeste. Presenta una longitud de $147 \mathrm{~km}$, y sus principales tributarios son el río Azufre y las quebradas de Caracarani, Colpitas, Hospicio, y los afluentes Putre y Socoroma. Con 3378 $\mathrm{km}^{2}$ de superficie, cubre parcialmente la provincia de Parinacota, donde el río nace a los 3900 msnm en el volcán Tacora, recorriendo la precordillera, el altiplano hasta desembocar en la costa de Arica (Figura 1). El uso de suelo en las nacientes (alta cordillera) está constituido principalmente por bofedales, mientras que en la parte baja de la cuenca 


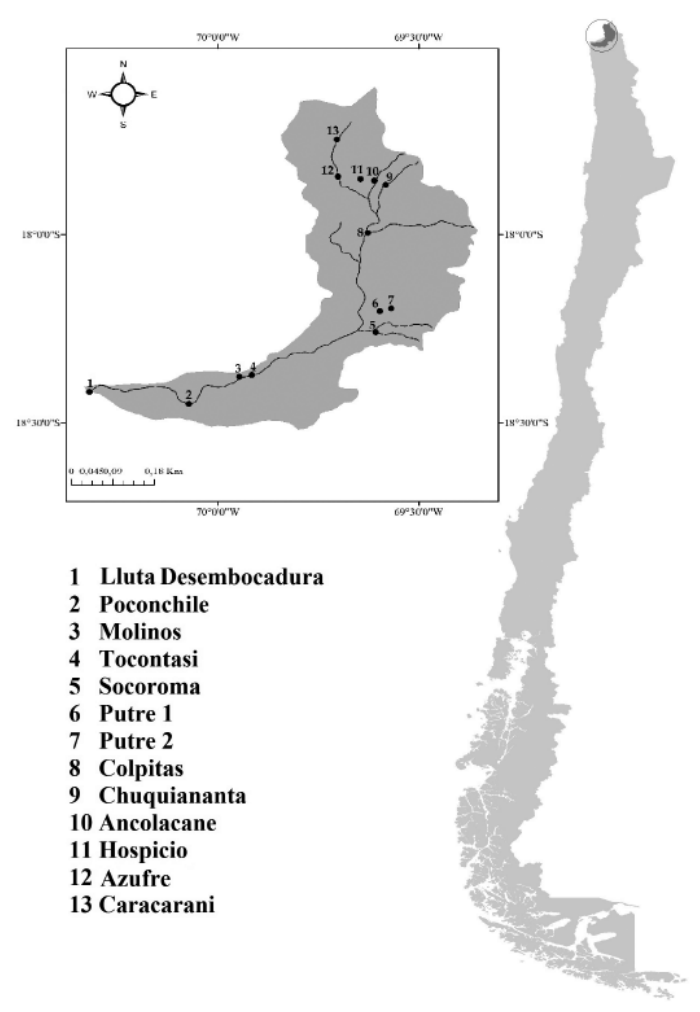

Figura 1: Área de estudio mostrando las 12 estaciones de muestreo presentes en la cuenca del río Lluta.

el uso principal es de terreno agrícola (maíz, alfalfa y hortalizas) $\left(76 \mathrm{~km}^{2}\right)$ (DGA 2004).

El clima en la región es templado, presentándose las mayores precipitaciones en el sector altiplánico $(237,7 \mathrm{~mm}$ de lluvia en temporada estival, producto del llamado invierno boliviano), mientras que en la parte baja de la cuenca, la media anual es de 0,7 mm (DGA 2004), ambas se presentarían en los meses de enero y febrero. La temperatura media anual registrada en el sector altiplánico es de $8,4^{\circ} \mathrm{C}$, mientras que en la parte baja es de $18,7^{\circ} \mathrm{C}$, siendo los meses más cálidos enero y febrero.

\section{Metodología}

\section{Muestreo}

Se realizó una campaña de muestreo desde el 23 hasta el 25 de septiembre de 2011 en 10 estaciones situadas a lo largo del sistema hídrico de la cuenca del río Lluta (Figura 1). En cada estación de muestreo se tomaron tres réplicas con una red surber $(0,09$ $\mathrm{m}^{2}, 200 \mu \mathrm{m}$ abertura de malla), dos en el sector de corriente y uno en la orilla privilegiando la presencia de vegetación. Las muestras fueron fijadas con alcohol a $95 \%$ y llevadas a laboratorio donde se procedió a su separación e identificación hasta el nivel taxonómico más bajo posible bajo una lupa estereoscópica con ayuda de literatura especializada. Posteriormente los macroinvertebrados fueron clasificados en seis grupos funcionales alimentarios (GFA): colector, filtrador, depredador, detritívoro, fragmentador y ramoneador, de acuerdo con los criterios de Merrit \& Cummins (1996) y Tomanova et al. (2006).

\section{Análisis de datos}

La estructura de la comunidad de macroinvertebrados fue analizada usando los índices ecológicos de Equidad de Pielou (J'), Índice de Diversidad de Shannon-Wiener (H') e Índice de dominancia de Berger-Parker (B').

Para poder determinar las relaciones entre las estaciones de muestreo basados en la distribución y abundancias de macroinvertebrados, los datos fueron primariamente transformados con raíz cuadrada, de manera de darles menos peso a los taxa más abundantes. Luego se construyó una matriz de similitud basándose en el índice de Bray \& Curtis, la que fue analizada utilizando el procedimiento de escalamiento multidimensional (nMDS - Clarke \& Gorley 2005) como método de ordenación, para visualizar las relaciones entre los grupos en un espacio multidimensional, evaluando representaciones de 2-dimensiones. Para analizar diferencias en la distribución de los macroinvertebrados entre las estaciones, nosotros usamos un análisis de similitud de 1-vía (ANOSIM - Clarke \& Gorley 2005), con las zonas (cordillera, precordillera, pampa y desembocadura) como factor.

Con el objeto de determinar las relaciones entre las estaciones, basados en la distribución y abundancias de los grupos funcionales alimentarios, nosotros usamos el mismo procedimiento antes descrito.

Todos estos análisis fueron llevados a cabo empleando el software PRIMER v.6-Plymouth Routines in Multivariate Ecological Research (Clarke et al. 2005).

\section{Resultados y Discusión}

Se registró un total de 66 taxa en la cuenca del río Lluta, pertenecientes a 17 órdenes y a 9 clases, 
correspondiendo la mayor parte a la clase Insecta, específicamente al orden Diptera con 20 morfo especies (Tabla 1), un número ostentosamente mayor a lo registrado para otras cuencas áridas y semiáridas cercanas, e.g. Loa - 15 taxa, Limarí - 46 taxa (Palma et al. 2013), Huasco - 43 taxa (Alvial et al. 2013) y para humedales semiáridos, e.g. humedales cercanos a la ciudad de Coquimbo - 42 taxa (Figueroa et al. 2009).
Las estaciones con mayores densidades de macroinvertebrados correspondieron a Molinos con 19.506 ind. $\mathrm{m}^{-2}$, Tocontasi con 14.104 ind. $\mathrm{m}^{-2}$ y Caracarani con 11.389 ind. $\mathrm{m}^{-2}$, mientras que la menor densidad fue la estación Ancolacane con 241 ind. $\mathrm{m}^{-2}$. Chironomidae (Diptera) correspondió a la familia más abundante en el área de estudio, registrándose la mayor abundancia de esta familia en la estación Molinos con 16.842 ind. $\mathrm{m}^{-2}$.

Tabla 1. Lista y densidad (ind. $\mathrm{m}^{-2}$ ) de especies de macroinvertebrados bentónicos acuáticos en las 12 estaciones de muestreo ubicadas en cuenca del río Lluta, Arica, registrados durante septiembre de 2011. Grupo funcional trófico (GFT) asignado: D, detritívoro; R, ramoneador; CF, colector filtrador; CR, colector recolector; P, predador; F, fragmentador; ?, desconocido. Estaciones de muestreo, Ll: Lluta desembocadura, Po: Poconchile, Mo: Molinos, To: Tocontasi, Pu1: Putre 1, Pu2: Putre 2, Co: Colpitas, Ch: Chuquiananta, An: Ancolacane, Ho: Hospicio, Az: Azufre, Ca: Caracarani.

\begin{tabular}{|c|c|c|c|c|c|c|c|c|c|c|c|c|c|}
\hline \multirow[b]{2}{*}{ Taxa } & \multirow[b]{2}{*}{ GFT } & \multicolumn{12}{|c|}{ Estaciones de muestreo } \\
\hline & & $L l$ & Po & Mo & To & Pul & Pu2 & Co & Ch & $A n$ & Ho & $A z$ & $\mathrm{Ca}$ \\
\hline \multicolumn{14}{|l|}{ Annelida } \\
\hline Oligochaeta sp. 1 & $\mathrm{D}$ & 0 & 166,5 & 0 & 0 & 0 & 0 & 0 & 0 & 0 & 140,6 & 0 & 0 \\
\hline Oligochaeta sp. 2 & $\mathrm{D}$ & 0 & 18,5 & 0 & 0 & 0 & 0 & 0 & 7,4 & 0 & 0 & 0 & 0 \\
\hline Naididae sp. & $\mathrm{D}$ & 0 & 0 & 0 & 1805,6 & 0 & 0 & 0 & 0 & 7,4 & 0 & 0 & 0 \\
\hline Arhynchobdellidae sp. & $\mathrm{D}$ & 0 & 14,8 & 0 & 0 & 0 & 0 & 0 & 0 & 0 & 144,3 & 0 & 0 \\
\hline Turbellaria sp. & $\mathrm{D}$ & 0 & 0 & 0 & 0 & 0 & 0 & 0 & 0 & 0 & 0 & 0 & 11,1 \\
\hline \multicolumn{14}{|l|}{ Mollusca } \\
\hline Biomphalaria aymara & $\mathrm{R}$ & 0 & 0 & 0 & 0 & 0 & 0 & 0 & 0 & 0 & 11,1 & 0 & 0 \\
\hline Physa sp. & $\mathrm{R}$ & 7,4 & 0 & 7,4 & 0 & 0 & 0 & 0 & 0 & 0 & 0 & 0 & 0 \\
\hline Melanoides maculata & $\mathrm{R}$ & 0 & 0 & 0 & 0 & 0 & 0 & 0 & 0 & 0 & 0 & 0 & 0 \\
\hline Littoridina sp. 1 & $\mathrm{R}$ & 825,1 & 4317,9 & 3,7 & 7,4 & 0 & 0 & 0 & 0 & 0 & 0 & 0 & 0 \\
\hline Littoridina sp. 2 & $\mathrm{R}$ & 14,8 & 3145 & 0 & 0 & 0 & 0 & 0 & 3,7 & 0 & 0 & 0 & 0 \\
\hline Bivalvia sp. & $\mathrm{CF}$ & 0 & 0 & 0 & 0 & 0 & 0 & 0 & 0 & 0 & 7,4 & 0 & 0 \\
\hline \multicolumn{14}{|l|}{ Malacostraca } \\
\hline Isopoda sp. & $\mathrm{CR}$ & 0 & 0 & 0 & 0 & 0 & 0 & 0 & 0 & 0 & 0 & 0 & 0 \\
\hline Hyalella sp. & $\mathrm{CR}$ & 11,1 & 0 & 0 & 0 & 0 & 0 & 0 & 0 & 18,5 & 303,4 & 0 & 10944,6 \\
\hline Cryphiops caementarius & $\mathrm{P}$ & 74 & 0 & 0 & 0 & 0 & 0 & 0 & 0 & 0 & 0 & 0 & 0 \\
\hline \multicolumn{14}{|l|}{ Archnida } \\
\hline Hygrobatidae sp. 1 & $\mathrm{P}$ & 0 & 7,4 & 0 & 11,1 & 0 & 0 & 0 & 22,2 & 0 & 0 & 0 & 3,7 \\
\hline Hygrobatidae sp. 2 & $\mathrm{P}$ & 0 & 0 & 0 & 0 & 0 & 0 & 0 & 0 & 11,1 & 3,7 & 0 & 0 \\
\hline Hydrachnidia sp. 1 & $\mathrm{P}$ & 0 & 0 & 7,4 & 0 & 0 & 0 & 0 & 3,7 & 0 & 22,2 & 0 & 7,4 \\
\hline Hydrachnidia sp. 2 & $\mathrm{P}$ & 0 & 48,1 & 0 & 3,7 & 0 & 0 & 0 & 48,1 & 0 & 7,4 & 0 & 3,7 \\
\hline Hydrachnidia sp. 3 & $\mathrm{P}$ & 0 & 0 & 0 & 0 & 0 & 0 & 0 & 0 & 0 & 0 & 0 & 0 \\
\hline \multicolumn{14}{|l|}{ Enthognatha } \\
\hline Collembola sp. & $\mathrm{CR}$ & 0 & 0 & 11,1 & 3,7 & 3,7 & 22,2 & 0 & 0 & 0 & 333 & 0 & 3,7 \\
\hline \multicolumn{14}{|l|}{ Coleoptera } \\
\hline Elmidae & $\mathrm{CR}$ & 0 & 277,5 & 1594,7 & 1028,6 & 0 & 3,7 & 62,9 & 1583,6 & 11,1 & 1883,3 & 111 & 7,4 \\
\hline Staphylinidae sp. 1 & $\mathrm{P}$ & 0 & 3,7 & 0 & 0 & 0 & 0 & 0 & 3,7 & 0 & 0 & 0 & 0 \\
\hline Staphylinidae sp. 2 & $\mathrm{P}$ & 0 & 3,7 & 0 & 0 & 0 & 0 & 0 & 0 & 0 & 0 & 0 & 0 \\
\hline Lancetes nigriceps & $\mathrm{P}$ & 3,7 & 7,4 & 7,4 & 59,2 & 0 & 0 & 0 & 14,8 & 0 & 0 & 0 & 0 \\
\hline Liodessus sp. & $\mathrm{P}$ & 0 & 159,1 & 0 & 0 & 0 & 0 & 0 & 0 & 0 & 7,4 & 0 & 0 \\
\hline Coleoptera sp. 1 & $?$ & 0 & 3,7 & 0 & 0 & 0 & 0 & 0 & 0 & 0 & 0 & 0 & 0 \\
\hline Coleoptera sp. 2 & $?$ & 0 & 0 & 0 & 0 & 0 & 0 & 7,4 & 0 & 0 & 0 & 0 & 0 \\
\hline \multicolumn{14}{|l|}{ Diptera } \\
\hline Empididae sp. 1 & $\mathrm{P}$ & 0 & 0 & 0 & 29,6 & 0 & 0 & 3,7 & 3,7 & 0 & 0 & 0 & 0 \\
\hline Empididae sp. 2 & $\mathrm{P}$ & 0 & 0 & 0 & 0 & 0 & 3,7 & 0 & 0 & 0 & 0 & 0 & 0 \\
\hline Ceratopogonidae sp. 1 & $\mathrm{P}$ & 0 & 29,6 & 40,7 & 37 & 0 & 18,5 & 11,1 & 33,3 & 3,7 & 44,4 & 37 & 0 \\
\hline Ceratopogonidae sp. 2 & $\mathrm{P}$ & 0 & 0 & 0 & 7,4 & 0 & 0 & 0 & 25,9 & 0 & 18,5 & 0 & 0 \\
\hline Ceratopogonidae sp. 3 & $\mathrm{P}$ & 0 & 0 & 0 & 0 & 0 & 0 & 0 & 0 & 0 & 7,4 & 0 & 0 \\
\hline Chironomidae sp. 1 & $\mathrm{CR}$ & 14,8 & 503,2 & 318,2 & 118,4 & 48,1 & 14,8 & 3,7 & 281,2 & 29,6 & 0 & 0 & 14,8 \\
\hline Chironomidae sp. 2 & $\mathrm{CR}$ & 270,1 & 329,3 & 15665,8 & 3022,9 & 14,8 & 62,9 & 11,1 & 381,1 & 18,5 & 577,2 & 0 & 0 \\
\hline Chironomidae sp. 3 & $\mathrm{CR}$ & 0 & 11,1 & 0 & 6830,2 & 55,5 & 181,3 & 59,2 & 22,2 & 0 & 1605,8 & 0 & 48,1 \\
\hline Chironomidae sp. 4 & $\mathrm{CR}$ & 0 & 0 & 0 & 0 & 0 & 0 & 11,1 & 3,7 & 7,4 & 33,3 & 14,8 & 159,1 \\
\hline Chironomidae sp. 5 & $\mathrm{CR}$ & 0 & 3,7 & 425,5 & 18,5 & 3,7 & 3,7 & 11,1 & 37 & 0 & 3,7 & 0 & 0 \\
\hline
\end{tabular}




\begin{tabular}{|c|c|c|c|c|c|c|c|c|c|c|c|c|c|}
\hline \multirow[b]{2}{*}{ Taxa } & \multirow[b]{2}{*}{ GFT } & \multicolumn{12}{|c|}{ Estaciones de muestreo } \\
\hline & & $L l$ & Po & Mo & To & Pul & $P u 2$ & Co & Ch & $A n$ & Ho & $A z$ & $\mathrm{Ca}$ \\
\hline Chironomidae sp. 6 & $\mathrm{CR}$ & 0 & 0 & 0 & 59,2 & 37 & 173,9 & 11,1 & 40,7 & 0 & 1324,6 & 462,5 & 3,7 \\
\hline Dolychopodidae & $\mathrm{P}$ & 0 & 3,7 & 0 & 0 & 0 & 0 & 0 & 0 & 0 & 0 & 0 & 0 \\
\hline Empididae sp. & $\mathrm{P}$ & 0 & 81,4 & 74 & 3,7 & 0 & 0 & 3,7 & 0 & 0 & 0 & 0 & 0 \\
\hline Ephydridae sp. & $\mathrm{CR}$ & 0 & 0 & 0 & 0 & 0 & 0 & 0 & 0 & 0 & 7,4 & 0 & 0 \\
\hline Tabanidae sp. & $\mathrm{P}$ & 0 & 48,1 & 33,3 & 107,3 & 3,7 & 48,1 & 11,1 & 114,7 & 18,5 & 7,4 & 7,4 & 0 \\
\hline Simuliidae sp. 1 & $\mathrm{CF}$ & 3,7 & 281,2 & 284,9 & 3,7 & 77,7 & 25,9 & 51,8 & 0 & 3,7 & 18,5 & 0 & 3,7 \\
\hline Simuliidae sp. 2 & $\mathrm{CF}$ & 0 & 37 & 0 & 185 & 395,9 & 96,2 & 22,2 & 0 & 0 & 0 & 0 & 0 \\
\hline Simulium tenuipes & $\mathrm{CF}$ & 0 & 0 & 0 & 0 & 0 & 0 & 0 & 0 & 0 & 0 & 0 & 0 \\
\hline Simulium philippii & $\mathrm{CF}$ & 0 & 0 & 0 & 0 & 0 & 0 & 0 & 0 & 0 & 0 & 0 & 0 \\
\hline Simulium escomeli & $\mathrm{CF}$ & 0 & 0 & 0 & 0 & 0 & 0 & 0 & 0 & 0 & 0 & 0 & 0 \\
\hline \multicolumn{14}{|l|}{ Ephemeroptera } \\
\hline Andesiops peruvianus & CR & 0 & 0 & 0 & 0 & 0 & 7,4 & 133,2 & 0 & 0 & 0 & 0 & 0 \\
\hline Andesiops sp. & CR & 0 & 0 & 166,5 & 22,2 & 103,6 & 159,1 & 233,1 & 0 & 0 & 11,1 & 3,7 & 51,8 \\
\hline Baetidae sp. & CR & 0 & 0 & 0 & 0 & 0 & 29,6 & 0 & 832,5 & 66,6 & 0 & 0 & 0 \\
\hline Andesiops torrens & CR & 0 & 0 & 0 & 0 & 0 & 0 & 0 & 0 & 0 & 0 & 0 & 0 \\
\hline \multicolumn{14}{|l|}{ Gripopterygidae } \\
\hline Notoperlopsis femina & $\mathrm{F}$ & 0 & 0 & 0 & 0 & 0 & 0 & 0 & 0 & 0 & 0 & 3,7 & 0 \\
\hline Gripopterygidae sp. 1 & $\mathrm{~F}$ & 0 & 0 & 0 & 3,7 & 0 & 3,7 & 0 & 3,7 & 0 & 0 & 0 & 0 \\
\hline Gripopterygidae sp. 2 & $\mathrm{~F}$ & 0 & 0 & 0 & 0 & 0 & 0 & 0 & 11,1 & 0 & 0 & 0 & 70,3 \\
\hline \multicolumn{14}{|l|}{ Trichoptera } \\
\hline Cailloma sp. & $\mathrm{P}$ & 0 & 0 & 0 & 0 & 0 & 0 & 0 & 0 & 0 & 0 & 0 & 0 \\
\hline Metrichia sp. & $\mathrm{R}$ & 0 & 477,3 & 858,4 & 728,9 & 3,7 & 133,2 & 0 & 492,1 & 0 & 162,8 & 0 & 0 \\
\hline Flintiella sp. & $?$ & 0 & 0 & 0 & 0 & 3,7 & 0 & 0 & 0 & 0 & 0 & 0 & 14,8 \\
\hline Oxyethira sp. & $\mathrm{R}$ & 0 & 0 & 0 & 0 & 0 & 0 & 0 & 0 & 0 & 0 & 0 & 14,8 \\
\hline Trichoptera sp. 1 & ? & 0 & 0 & 0 & 0 & 0 & 0 & 11,1 & 0 & 0 & 18,5 & 0 & 25,9 \\
\hline Trichoptera sp. 2 & ? & 0 & 0 & 0 & 0 & 0 & 0 & 0 & 0 & 0 & 3,7 & 0 & 0 \\
\hline Trichoptera sp. 3 & ? & 0 & 0 & 0 & 0 & 7,4 & 0 & 0 & 0 & 0 & 0 & 0 & 0 \\
\hline \multicolumn{14}{|l|}{ Odonata } \\
\hline Enallagma sp. & $\mathrm{P}$ & 7,4 & 0 & 0 & 0 & 0 & 0 & 0 & 0 & 0 & 18,5 & 0 & 0 \\
\hline Aeshnidae sp. & $\mathrm{P}$ & 0 & 0 & 7,4 & 3,7 & 0 & 0 & 7,4 & 0 & 0 & 0 & 0 & 0 \\
\hline Aphididae sp. & $?$ & 0 & 3,7 & 0 & 3,7 & 0 & 0 & 0 & 0 & 0 & 0 & 0 & 0 \\
\hline \multicolumn{14}{|l|}{ Hemiptera } \\
\hline Saldidae sp. & $\mathrm{P}$ & 0 & 0 & 0 & 0 & 0 & 0 & 0 & 0 & 0 & 0 & 0 & 0 \\
\hline Corixidae sp. & $\mathrm{P}$ & 0 & 0 & 0 & 0 & 0 & 0 & 0 & 0 & 44,4 & 166,5 & 0 & 0 \\
\hline
\end{tabular}

Los parámetros comunitarios mostraron que la mayor cantidad de taxa se registró en la estación Hospicio con 28 taxa, mientras que lo contrario ocurrió en la estación Azufre con 7 taxa. Respecto del índice de equidad de Pielou este varió entre 0,09 y 0,86 para las estaciones Caracarani y Ancolacane. Los mayores valores de diversidad de Shannon fueron registrados en la estación Putre 2 con 2,27, mientras que los menores valores de diversidad se registraron en la estación Caracarani con 0,25. Los valores de dominancia de Berger-Parker fueron concordantes con los altos valores de Diversidad, registrándose la menor dominancia $(0,18)$ en la estación Putre 2, mientras que en la estación Caracarani se registró una alta dominancia $(0,96)$ dada por la alta abundancia de la especie Hyalella sp. con 10.945 ind.m ${ }^{-2}$ (Tabla 2).

El análisis de escalamiento multidimensional (nMDS) de las abundancias de macroinvertebrados bentónicos reveló seis grupos distintos a una similitud de $30 \%$. Un grupo estuvo compuesto por
Tabla 2. Número de especies (S), número de individuos (N), Índice de Equidad de Pielou (J'), Índice de Diversidad de Shannon-Wiener (H') e Índice de dominancia de Berger-Parker, registradas en las 12 estaciones de muestreo en la cuenca del río Lluta, Arica.

\begin{tabular}{lrrrcc}
\hline & S & N & J' & H' & D \\
\hline Lluta desembocadura & 10 & 1232 & 0,44 & 1,02 & 0,67 \\
Poconchile & 25 & 9983 & 0,51 & 1,66 & 0,43 \\
Molinos & 16 & 19506 & 0,30 & 0,83 & 0,80 \\
Tocontasi & 24 & 14104 & 0,49 & 1,55 & 0,48 \\
Putre 1 & 13 & 1691 & 0,47 & 1,21 & 0,61 \\
Putre 2 & 17 & 988 & 0,80 & 2,27 & 0,18 \\
Colpitas & 18 & 666 & 0,73 & 2,10 & 0,35 \\
Chuquiananta & 22 & 3970 & 0,59 & 1,83 & 0,40 \\
Ancolacane & 12 & 241 & 0,86 & 2,14 & 0,28 \\
Hospicio & 28 & 6893 & 0,62 & 2,06 & 0,27 \\
Azufre & 7 & 640 & 0,46 & 0,90 & 0,72 \\
Caracarani & 17 & 11389 & 0,09 & 0,25 & 0,96 \\
\hline
\end{tabular}

solo la estación de desembocadura (mayormente representados por Littoridina sp. 1), el segundo grupo incluyó a todas las estaciones de meseta y 
dos estaciones de la cordillera (8 y10) (mayormente representados por Elmidae y Chironomidae sp. 2), el tercer grupo incluyó a todas las estaciones ubicadas en la pampa (presentando una alta abundancia de Elmidae y Chironomidae sp.2), y finalmente los tres subgrupos fueron las estaciones de la cordillera (Representados principalmente por Chironomidae sp. 6, Baetidae sp. 1 y el crustáceo Hyalella). En general, los grupos Desembocadura, Pampa, Precordillera y Cordillera fueron estadísticamente significativos (ANOSIM, Global $\mathrm{R}=0,34, p=$ 0,02) (Figura 3).

Las estaciones ubicadas en el área de pampa y precordillera no registraron taxa exclusivos (Tabla 3), mientras que cuatro taxa fueron registrados solamente en las estaciones de cordillera (Turbellaria, Pulmonata, Bivalvia y Hemiptera) y solo el Decapoda Cryphiops caementarius fue colectado en la estación de desembocadura, alcanzando además altas abundancias. Este "camarón de río del norte" ha sido reportado en varias cuencas del norte de Chile, incluida esta cuenca en estudio (Jara et al. 2006), en donde además, se ha descrito que tiene una gran dispersión dentro de estas, registrándose desde las cabeceras hasta las desembocaduras, siendo en esta última donde ocurre la eclosión de los huevos y donde se han registrado grandes poblaciones en estados tempranos de desarrollo (Meruane et al. 2006).

La altitud ha sido demostrada como una variable importante en determinar los modelos de variación en los macroinvertebrados, debido a que esta se
Tabla 3. Macroinvertebrados colectados en cada área de muestreo en la cuenca del río Lluta, Arica.

\begin{tabular}{|c|c|c|c|c|}
\hline & Desembocadura & Pampa & Precordillera & Cordillera \\
\hline Oligochaeta & & $\mathrm{x}$ & & $\mathrm{x}$ \\
\hline Haplotaxida & & $\mathrm{x}$ & & $\mathrm{x}$ \\
\hline Hirudinea & & $\mathrm{X}$ & & $\mathrm{X}$ \\
\hline Turbellaria & & & & $\mathrm{X}$ \\
\hline Pulmonata & & & & $\mathrm{x}$ \\
\hline Bassomatophora & $\mathrm{X}$ & $\mathrm{X}$ & & \\
\hline Mesogastropoda & $\mathrm{X}$ & $\mathrm{X}$ & & $\mathrm{X}$ \\
\hline Bivalvia & & & & $\mathrm{X}$ \\
\hline \multicolumn{5}{|l|}{ Isopoda } \\
\hline Amphipoda & $\mathrm{x}$ & & & $\mathrm{x}$ \\
\hline Decapoda & $\mathrm{x}$ & & & \\
\hline Trombodiforma & & $\mathrm{X}$ & & $\mathrm{X}$ \\
\hline Collembola & & $\mathrm{X}$ & $\mathrm{x}$ & $\mathrm{X}$ \\
\hline Coleoptera & $\mathrm{X}$ & $\mathrm{X}$ & $\mathrm{x}$ & $\mathrm{x}$ \\
\hline Diptera & $\mathrm{X}$ & $\mathrm{X}$ & $\mathrm{X}$ & $\mathrm{X}$ \\
\hline Ephemeroptera & & $\mathrm{X}$ & $\mathrm{x}$ & $\mathrm{X}$ \\
\hline Plecoptera & & $\mathrm{X}$ & $\mathrm{x}$ & $\mathrm{x}$ \\
\hline Trichoptera & & $\mathrm{X}$ & $\mathrm{x}$ & $\mathrm{X}$ \\
\hline Odonata & $\mathrm{x}$ & $\mathrm{X}$ & $\mathrm{x}$ & $\mathrm{X}$ \\
\hline Hemiptera & & & & $\mathrm{x}$ \\
\hline
\end{tabular}

relaciona a diferentes factores como cambios en la temperatura o vegetación ribereña a lo largo de un río (Jacobsen et al. 1997). Nuestros resultados permitieron observar una zonación en la comunidad de macroinvertebrados desde la cabecera de la cuenca hasta la desembocadura en el mar, sin embargo, esta no siguió los patrones descritos por el concepto de río continuo -Vannote et al. 1980- para los grupos funcionales alimentarios, debido a que gran parte de la cuenca del río Lluta estuvo bien representada
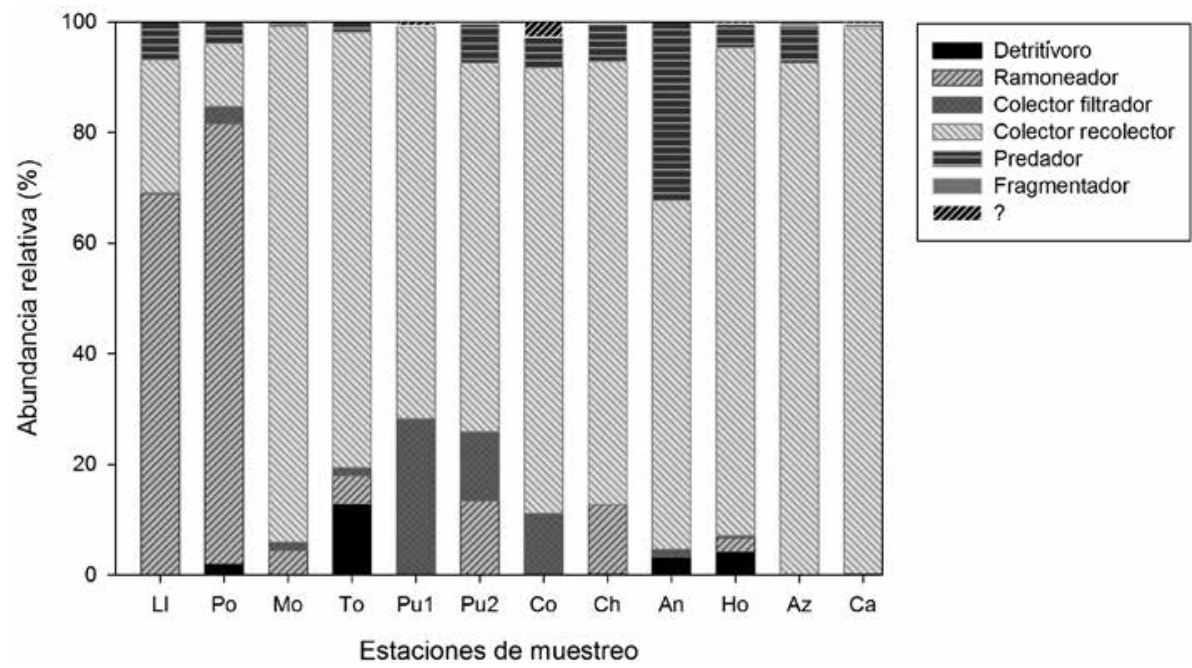

Figura 2: Abundancia relativa de los grupos funcionales tróficos en la cuenca del río Lluta. 
por el grupo de colectores-recolectores, dominando en 10 de las 12 estaciones ( 3 a 12), mientras que el grupo de los ramoneadores dominaron en las dos estaciones restantes, siendo estas las de menor altura (1 desembocadura; 2 meseta) (Figura 2). En conjunto no hubo diferencia significativa entre los grupos (ANOSIM, Global $\mathrm{R}=0,26, p=0,08$ ).

De los 66 taxa identificados en las muestras, 5 correspondieron a detritívoros, 7 a ramoneadores, 6 a colectores-filtradores, 15 a colectoresrecolectores, 23 a depredadores, 3 a fragmentadores y 7 especies fueron indeterminadas. Los colectoresrecolectores han sido reportados como los grupos más frecuentes en ríos del hemisferio sur (Chará-Serna et al. 2010, Miserendino \& Pizzolon 2003), los que se alimentan de materia orgánica particulada $(<1 \mathrm{~mm})$, la que probablemente en este estudio tuvo su origen en la vegetación ribereña, principalmente por humedales del tipo "bofedal", tipo vegetacional natural siempreverde, cuyo estrato herbáceo se desarrolla en cojines emergentes sobre los niveles de inundación (Castellaro et al. 2004). Por su parte, individuos con hábitos de alimentación fragmentadora estuvieron escasamente representados en este estudio. Estos resultados son similares a los registrados en la cuenca del río Loa por Palma et al. (2013), debido posiblemente a la escasa materia orgánica gruesa $(>1 \mathrm{~mm})$ importada hacia el río. Los ramoneadores dominaron en las estaciones más bajas altitudinalmente (Lluta desembocadura y Poconchile), siendo su principal fuente de alimento las algas bentónicas (CharáSerna et al. 2010), esto fue corroborado por nosotros en el área de estudio, donde ambas estaciones de muestreo presentaron una gran cantidad de algas. Es importante mencionar en este trabajo que la estación Lluta desembocadura presenta una importante actividad agrícola, comparada con las demás estaciones, la que se encuentra acompañada además del uso de químicos tanto para el control de plagas como del abono del suelo para la diversidad de cultivos que allí se desarrollan (Torres \& Acevedo 2008), posibles factores que explicarían la baja diversidad de macroinvertebrados encontrada.

\section{Conclusiones}

Nuestros resultados indicaron una zonación de macroinvertebrados desde la cabecera de la cuenca

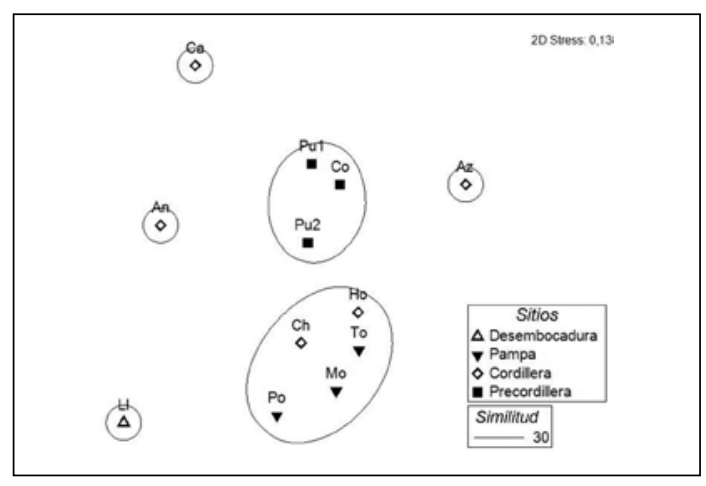

Figura 3: Visualización del ensamble de macroinvertebrados basado en un análisis de escalamiento multidimensional (nMDS). Los grupos están caracterizados por las zonas de distribución en el río Lluta.

hasta la desembocadura en la cuenca del río Lluta. Siendo el grupo mejor representado la familia Chironomidae, mientras que el grupo funcional de los colectores-recolectores dominaron en 10 de las 12 estaciones, en tanto en las otras dos estaciones (las más bajas en altura) estuvieron dominadas por individuos ramoneadores (principalmente especies de gastrópodos del genero Littoridina).

La mayor parte de los invertebrados acuáticos no han sido importantes cuando se trata de estimar el estado de conservación de las especies de Chile. Esto debería cambiar cuando las instancias competentes asuman el importante rol que ellos cumplen en el ecosistema que integran y la declinación que están sufriendo sus poblaciones. Estos representan una parte integral de sistemas lóticos tratando materia orgánica y energía que provee a los niveles tróficos más altos, por lo tanto, un entendimiento de los efectos de estresantes antropogénicos y naturales, sobre la distribución y abundancia es crítico para la evaluación del completo impacto en los sistemas fluviales.

\section{Agradecimientos}

Se agradece en primera instancia al Ministerio de Medio Ambiente de Arica y Parinacota por la autorización de publicar estos datos, a Margarita Ruiz de Gamboa y Andrés Taucare por participar de los terrenos en proceso de las muestras y datos.

Estudio financiado por licitación "12556-5L111" del Ministerio de Medio Ambiente de Arica y Parinacota. 


\section{Literatura Citada}

Alvial, I.; Orth, K.; Durán, B.; Álvarez, E.; Squeo, F.

2013. Importance of geochemical factors in determining distribution patterns of aquatic invertebrates in mountain streams south of the Atacama Desert, Chile. Hydrobiologia, 709(1): 11-25.

Campos Ortega, H.; Diaz Muños, G.; Campos Ortega, C. 2007. Aportes sedimentarios de los ríos Lluta y San José en la zona costera de la rada de Arica, Chile. Idesia, 25(2): 37-48.

Castellaro, G.; Ullrich, T.; Wackwitz, B.; Raggi, A.

2004. Composición botánica de la dieta de alpacas (Lama pacos L.) y llamas (Lama glama L.) en dos estaciones del año, en praderas altiplánicas de un sector de la Provincia de Parinacota, Chile. Agricultura Técnica, 64(4): 353-364.

Chará-Serna, A.; Chará, J.; Zuñiga, M.D.C.; Pedraza, G.;

Giraldo, L.

2010. Clasificación trófica de insectos acuáticos en ocho quebradas protegidas de la ecorregión cafetera colombiana. Universitas Scientiarium, 15(1): 1-11.

Clarke, K.R.; Gorley, R.N.

2005. PRIMER v.6: User Manual/Tutorial. PRIMER-E Ltda., Playmouth, UK. $192 \mathrm{p}$

Clarke, K.R.; Warwick, R.M.; Somerfield, P.J.; Gorley, R.N.

2014. Change in marine communities: an approach to statistical analysis and interpretation, 3rd edn. PRIMER-E Ltda, Plymouth, UK. 260 p.

Dirección General de Aguas (DGA)

2004. Diagnóstico y clasificación de los cursos y cuerpos de agua según objetivos de calidad. Cuenca del río Lluta. 99 p.

Fierro, P.; Bertrán, C.; Mercado, M.; Peña-Cortés, F.; Tapia, J.;

Hauenstein, E.; Vargas-Chacoff, L.

2012. Benthic macroinvertebrates assemblages as indicators of wáter quality applying a modified biotic index in a spatioseasonal context in a coastal basin of southern Chile. Revista de Biología Marina y Oceanografía, 47(1): 23-33.

Fierro, P.; Bertran, C.; Martinez, D.; Valdovinos, C.; VargasChacoff, L.

2014. Ontogenetic and temporal changes in the diet of the Chilean silverside Odontesthes regia (Atherinidae) in southern Chile. Cahiers de Biologie Marine, 55(3): 323-332.

Figueroa, R.; Suarez, M.; Andreu, A.; Ruiz, V.

2009. Caracterización ecológica de humedales de la zona semiárida en Chile central. Gayana, 73(1): 76-94.

Hynes, $\mathrm{H}$.

1970. The ecology of running waters. Uniersity of Toronto Press, Ontario, Canadá. 555 p.

Jacobsen, D.; Schultz, R.; Encalada, A.

1997. Structure and diversity of stream invertebrate assemblages: the influence of temperatura with altitude and latitude. Freshwater Biology, 38(2): 247-261.

Jara, C.; Rudolph, E.; González, E.

2006. Estado de conocimiento de los malacostráceos dulceacuicolas de Chile. Gayana, 70 (1): 40-49.
Merrit, R.W. And Cummins, K.W.

1996. Trophic relations of macroinvertebrates. En: Methods in Stream Ecology. Hahuer, R.F. and Lamberti, G.A. San Diego, CA, pp. 453-473.

Meruane, J.; Morales, M.; Galleguillos, C.; Rivera, M.; Hosokawa, $\mathrm{H}$.

2006. Experiencias y resultados de investigaciones sobre el camaron de río del Norte Cryphiops caementarius (Molina 1782) (Decapoda: Palaemonidae): Historia natural y cultivo. Gayana, 70(2): 280-292.

Miserendino M.L.; Pizzolon, L.A.

2003. Distribution of macroinvertebrate assemblages in the Azul-Quemquemtreu river basin, Patagonia, Argentina. New Zealand Journal of Marine and Freshwater Research, 37(3): 525-539.

Molina, C.; Gibon, F.M.; Pinto, J.; Rosales, C.

2008. Estructura de macroinvertebrados acuáticos en un río altoandino de la cordillera real, Bolivia: Variación anual y longitudinal en relación a factores ambientales. Ecología Aplicada, 7(1, 2): 105-116.

Morrone, J.J.

2006. Biogeographic areas and transition zones of Latin America and the Caribbean Islands based on panbiogeographic and cladistic analyses of the entomofauna. Annual Review of Entomology, 51: 467-494.

Palma, A.; González-Barrientos, J.; Reyes, C.; Ramos-Jiliberto, R. 2013. Biodiversidad y estructura comunitaria de ríos en las zonas árida, semiárida y mediterránea-norte de Chile. Revista Chilena de Historia Natural, 86(1): 1-14.

Parada, E. And S. Peredo.

2006. Estado de conocimiento de los bivalvos dulceacuícolas de Chile. Gayana, 70(1): 82-87.

Tomanova, S.; Goitia, E.; Helesic, J.

2006. Trophic levels and functional feeding groups of macroinvertebrates in Neotropical streams. Hydrobiologia, 556(1): 251-264.

Torres, A. And Acevedo, E.

2008. El problema de salinidad en los recursos suelo y agua que afectan el riego y cultivos en los valles de Lluta y Azapa en el norte de Chile. Idesia, (Chile), 26(3): 31-44.

Valdovinos, $\mathrm{C}$.

2006. Invertebrados dulceacuícolas. En: Biodiversidad de Chile. Patrimonios y desafíos. Conama. Ed. Ocho Libros. Santiago, Chile, pp. 204-225.

Vannote, R.L.; Minshall, G.W., Cummins, K.W.; Sedell, J.R.; Cushing, C.E.

1980. The River Continuum Concept. Canadian Journal of Fisheries \& Aquatic Research, 37: 130-137.

Yixing, Y.; Youpeng, X.; Ying, C.

2012. Relationship between changes of river-lake networks and water levels in typical regions of Taihu lake basin, China. Chinese Geographical Science, 22(6): 673-682. 